\title{
i-Generalized Homeomorphisms and Generalized i- Homeomorphisms in Topological Spaces
}

\author{
Sabih W. Askandar \\ Department of Mathematics/College of Education For Pure Science \\ University of Mosul \\ Mosul-Iraq \\ sabeehqaqus@yahoo.com \\ Received \\ 01/03/2018 \\ Accepted \\ 06/05/2018
التثاكلات المعمة من النوع-i والتثاكلات من النوع-i المعمة في
الفضاءات التبولوجية
م.م. صبيح وديع اسكندر \\ قسم الرياضيات/كلية التربية للعلوم الصرفة/جامعة الموصل

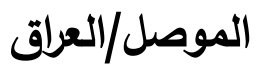

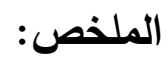

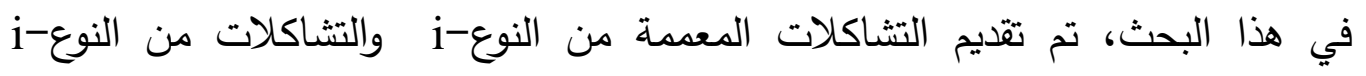

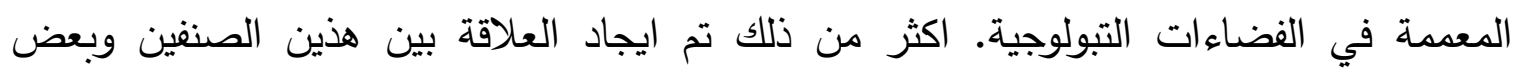 \\ الاصناف الاخرى من التثاكلات التبولوجية. \\ الكلمات المفتاحية: التثاكل التبولوجي.
}

\section{ABSTRACT:}

In this paper, topological ig-Homeomorphisms and topological giHomeomorphisms are introduced. Further, the relations between these two classes and some other classes of Homeomorphisms are investigated.

Keywords: Topological Homeomorphism

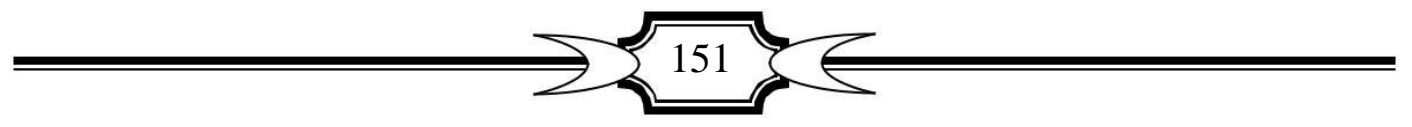




\section{INTRODUCTION:}

In 1970, Levine [7], has generalized the concept of closed sets to generalized closed sets [1]. In 1987, Bhattacharyya and Lahiri [3], generalized the concept of closed sets to semi-generalized closed sets with the help of semi-open sets and they obtaining various topological properties. In 1990, Arya and Nour [1], defined generalized semi-open sets with the help of semi-openness and used them to obtain some characterizations of snormal spaces. In 1995, Devi, Balachandran and maki [0], defined two classes of maps that are called semi-generalized homeomorphisms and generalized semi-homeomorphisms. In 2006, Rajesh, N., Ekici, E. and Thivagar, M. L.[9], introduced a new class of homeomorphisms called, gs*_ homeomorphisms. In 2009, Caldas, M., Jafari, S. and Rajesh, N. [4], introduced $\tilde{g}$-homeomorphisms. In 2012, Mohammed, A.A. and Askandar, S.W., [8][2], introduced the concept of i-open sets. In this paper we generalize the concept of closed sets to i-generalized closed sets with the help of i-open sets and obtained various topological properties. We introduce two classes of maps are called ig-homeomorphisms and gi-homeomorphisms and we study their properties. Throughout this $\operatorname{paper}(X, \tau)$ and $(Y, \delta)$ are always topological spaces, $f$ is always a mapping $\operatorname{from}(X, \tau)$ into $(Y, \delta)$ and $C l(A)$ denotes the closure of a set $A$.

\section{Definitions and Examples.}

In this Section, we introduce important new concepts of sets and maps and use them to prove the main results. $A \subseteq X$ is called i-open set [2][8] if $A \subseteq C l(A \cap G)$ (for some $\mathrm{G} \neq \phi, \mathrm{X}$ is open set in $X . X / A$ is called $\mathrm{i}$-closed set [2][8]. For example if $X=\{1,2,3\}, \tau=\{\phi,\{1\},\{1,3\}, X\}$, we have: Closed sets which are: $X,\{2,3\},\{2\}, \phi$, i-open sets are : $\phi,\{1\},\{1,3\},\{3\},\{1$, $2\},\{2,3\}, X$. i-closed sets are: $X,\{2,3\},\{2\},\{1,2\},\{3\},\{1\}, \phi$.

Lemma1.1. Every open set is i-open. But the converse is not necessary to be trrue [8][2].

Corollary1.2. Every closed set is i-closed. But the converse is not necessary to be trrue [8][2].

Definition1.3. Let $\tau^{\mathrm{i}}$ be the set of all i-open sets of $X$ and let $A \subseteq \mathrm{X}$ then:

1. $C l_{i}(A)=\bigcap_{o \in A} F_{i} . A \subseteq F_{i} \forall i$ (Where $F_{i} \subseteq X$ is i-closed set $\forall i$ and $C l_{i}(A)$ is the i-closure of $A[8][2] . A=C l_{i}(A)$ if and only if $A$ is i-closed set. 
2. $\operatorname{Int}_{i}(A)=\bigcup_{i \in \Lambda} I_{i} I \subseteq A \forall i$. (Where $I_{\mathrm{i}} \subseteq X$ is i-open set $\forall i$ and $\operatorname{Int}_{i}(A)$ is $\mathrm{i}$ interior of $A[8][2]$.

Definition1.4. A subset $A$ of $X$ is said to be:

1. generalized closed set ( $g$-closed ) [1] if $C l(A) \subseteq U$ where $A \subseteq U$ and $U \subseteq X$ is open set. $B \subseteq X$ is generalized open set ( $g$-open )[1] if its complement is $g$-closed.

2. generalized $i$-open set ( gi-open ) if $F \subseteq \operatorname{Int}_{i}(A)$,

Where, $F \subseteq A \subseteq X$ is closedset. $B \subseteq X$ is generalized $i$-closed set ( gi-closed ) if its complement is gi-open.

3. $i$-generalized closed set (ig-closed ) if $C l_{i}(A) \subseteq U$ where $A \subseteq U$ and $U \subseteq X$ is an $i$-open set. $B \subseteq X$ is i-generalized open set (ig-open) if its complement is ig-closed.

Definition1.5. i-Generalized Closure of $A$, denoted by $C l_{i g}(A)$ : $C l_{i g}(A)=\bigcap_{i \in A} F_{i} \cdot A \subseteq F_{i} \quad \forall i$ Where $F_{i} \subseteq X$ is ig-closed set $\forall i, A=C l_{i g}(A)$ if and only if $A$ is ig-closed set.

Example1.6. Let $X=\{4,5,6\}, \tau=\{\phi,\{4\}, X\}$.

Therefore, Closed sets are: $X,\{5,6\}, \phi$. i-open sets are : $\phi,\{4\},\{4,5\},\{4,6\}, X, i$-closed sets are: $X,\{5,6\},\{6\},\{5\}, \phi$. gi-open sets are: : $\phi,\{4\},\{5\},\{6\},\{4,5\},\{4,6\}, X$, gi-closed sets are: $X,\{5,6\},\{4,6\},\{4,5\},\{6\},\{5\}, \phi$. ig-closed sets are: $\phi,\{5\},\{6\},\{5,6\}, X$, ig-open sets are: $X,\{4,6\},\{4,5\},\{4\}, \phi$.

Example1.7. Let $X=\{7,8,9\}, \tau=\{\phi,\{7\},\{7,8\},\{7,9\}, X\}$.

Therefore, Closed sets are: $X,\{8,9\},\{9\},\{8\}, \phi$. i-open sets are : $\phi,\{7\},\{8\},\{9\},\{7,8\},\{7,9\}, X$, i-closed sets are: $X,\{8,9\},\{7,9\},\{7,8\},\{9\},\{8\}, \phi$. gi-open sets are: : $\phi,\{7\},\{8\},\{9\},\{7,8\},\{7,9\},\{8,9\}, X$, gi-closed sets are: $X,\{8,9\},\{7,9\},\{7,8\},\{9\},\{8\},\{7\}, \phi$. ig-closed sets are: $\phi,\{7\},\{8\},\{9\},\{7,8\},\{7,9\},\{8,9\}, X$, ig-open sets are: $X,\{8,9\},\{7,9\},\{7,8\},\{9\},\{8\},\{7\}, \phi$.

Theorem1.8. Every i-closed set is i-generalized-closed. [6] 
Proof: Let $A \subseteq X$ be i-closed. Then $A=C l_{i}(A)$ It is clear that $A \subseteq C l_{i}(A)$ and $C l_{i}(A) \subseteq A$.

Suppose that $A \subseteq U$ and $U \subseteq X$ are i-open. Therefore, $C l_{i}(A) \subseteq U$. Hence $A$ is i-generalized closed. Also since $A \subseteq C l_{i g}(A) \subseteq C l_{i}(A) \subseteq C l(A)$ we have, every closed and i-closed sets are i-generalized-closed (where $C l(A)$ denotes the closure of a set $A$ ).

In (example 1.7) it is clear that $A=\{7\}$ is i-generalized closed, $A$ is not iclosed.

Theorem1.9. Every i-generalized closed set is generalized i-closed.[6] Proof: suppose that $A \subseteq X$ is i-generalized closed. We have, $C l_{i}(A) \subseteq U$ where $A \subseteq U$ and $U \subseteq X$ is i-open. Now $\left(C l_{i}(A) \subseteq U\right)^{C}=U^{C} \subseteq \operatorname{Int}_{i}\left(A^{C}\right)$, we have $U^{C}$ is i-closed set in $X$, we have $A^{C}$ is generalized i-open. Hence, $A$ is generalized i-closed.

In (example 1.6) $A=\{4,5\}$ is gi-closed set but it is not ig-closed.

Corollary1.10. Every closed set (respectively i-closed, ig-closed set) is giclosed. But the converse is not necessary to be true (see corollary (1.2.), theorem (1.8) and theorem (1.9)).

Definition1.11. A mapping $f$ is said to be:

1. Closed mapping (resp. open mapping) if $f(F)$ is closed set (resp. open set) in Y For each closed set(resp. open set) $F$ in $X$.

2. i-closed mapping (resp. i-open mapping) if the $f(F)$ is i-closed (resp. iopen) in $Y[8][2]$ for each closed set (resp. open set) $F$ in $X$.

3. i-generalized closed mapping (resp. i-generalized open mapping) if $f(F)$ i-generalized closed (resp. i-generalized open set) in $Y$ for each closed set (resp. open set) $F$ in $\mathrm{X}$.

4. Generalized i-open mapping (resp. generalized i-closed mapping) if $f(F)$ is gi-open (resp. gi-closed set) in $Y$ for each open set (resp. closed set) $F$ in $X$.

Definition1.12. A mapping $f$ is said to be:

1. Continuous if and only if the inverse of $I^{*}$ is closed (resp. open set) in $X$ for all closed (resp. open sets) $\mathrm{I}^{*}$ in $Y$.

2. i-continuous [8] [2] if and only if the inverse of $I^{*}$ is i-closed (resp. i-open set) in $X$ for every closed (resp. open sets) $I^{*}$ in $Y$.

3. i-generalized continuous if and only if the inverse of $I^{*}$ is ig-closed (resp. ig-open set) in $X$ for all closed (resp. open sets) $I^{*}$ in $Y$. 
4. Generalized i-continuous if and only if the inverse of $I^{*}$ is gi-closed (resp. gi-open set) in $X$ for all closed (resp. open sets) $I^{*}$ in $Y$.

5. i-generalized irresolute (in short, ig-irresolute) if and only if the inverse of $I^{*}$ is ig-closed set in $X$ for all ig-closed sets $I^{*}$ in $Y$.

6. Generalized i-irresolute (in short, gi-irresolute) if and only if the inverse of $I^{*}$ is gi-closed set in $X$ for all gi-closed sets $I^{*}$ in $Y$.

Definition1.13. A mapping $f$ is said to be:

i) Homeomorphism, if $f$ is (1) continuous, (2) open.

ii) i-Homeomorphism [8] [2] if $f$ is (1) i-continuous. (2) i-open.

iii) i-generalized Homeomorphism (in short igh) if $f$ is (1) ig-continuous,

(2) ig-open.

iv) Generalized i-Homeomorphism (in short gih) if $\boldsymbol{f}$ is (1) gi-continuous, (2) gi-open.

\section{The Main Results.}

In this section we find the relation between the new concepts of mappings.

Theorem2.1. Every open-mapping is i- open. [8][2]

Example2.2. Let $\mathrm{X}=\{10,11,12\}, \quad \tau=\{\phi,\{10,11\}, X\}, \quad \mathrm{Y}=\{10,11,12\}$, $\delta=\{\phi,\{11\},\{10,11\}, Y\}, f(10)=11, f(11)=12 f(12)=10, \delta^{\mathrm{i}}=\{\phi,\{11\},\{10$, $11\},\{10\},\{10,12\},\{11,12\}, Y\} . f$ is not open mapping because $\{10,11\}$ is open set in $X, f(\{10,11\})=\{11,12\}$ is not open set in $Y$. Hence $f$ is i-open mapping.

Corollary2.3. Every closed-mapping is i- closed.[8][2]

In (example 2.2) $f$ is not closed mapping because $\{12\}$ is closed set in $\mathrm{X}$ but $f(\{12\})=\{10\}$ is not closed set in $Y$. Hence $f$ is i-closed mapping.

Theorem2.4. Every i-closed mapping is i-generalized closed.

Proof: Suppose that $f$ is i-closed mapping. Then by suppose, $f(F)$ is iclosed set in $Y$ for each closed set $F$ in $X$. Then $f(F)$ is ig-closed set in $Y$ (theorem (1.8)). Hence $f$ is ig-closed.

Example2.5. Let $X=Y=\{13,14,15\}, \tau=\{\phi,\{13\},\{13,14\},\{13,15\}, X\}=\delta$, $f(13)=14, f(14)=13, f(15)=15, f$ is not $\mathrm{i}$-closed because $\{14,15\}$ is closed set in $X, f(\{14,15\})=\{13,15\}$ is not $\mathrm{i}$-closed in $Y$. Hence $f$ is ig-closed.

Corollary 2.6. Every i-open mapping is ig- open.

In (example 2.5) $f$ is not i-open because $\{13,15\}$ is open set in $X, f$ $(\{13,15\})=\{14,15\}$ is not $\mathrm{i}$-open set in $Y$. Hence $f$ is ig-open.

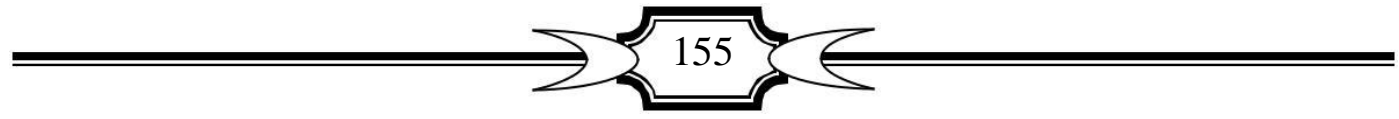


Theorem2.7. Every ig-closed mapping is gi-closed.

Proof: Suppose that $f$ be ig-closed mapping, $\mathrm{F}$ be a closed set in $\mathrm{X}$, then $f(F)$ is ig-closed in $Y$ (by suppose). $f(F)$ is gi-closed in $Y$ (theorem 1.9). Hence $f$ is gi-closed.

Example2.8. Let $\mathrm{X}=\mathrm{Y}=\{\mathrm{p}, \mathrm{q}, \mathrm{r}\}, \tau=\{\phi,\{\mathrm{p}\}, \mathrm{X}\}=\delta, f(p)=q, f(q)=p, f(r)=r, f$ is not ig-closed mapping because $\{\mathrm{q}, \mathrm{r}\}$ is closed in $X, f(\{\mathrm{q}, \mathrm{r}\})=\{\mathrm{p}, \mathrm{r}\}$ is not ig-closed in $Y$. Hence $f$ is gi-closed.

Corollary2.9. Every ig-open mapping is gi- open.

In (example 2.8) $f$ is not ig-open because $\{p\}$ is open in $X, f(\{p\})=\{q\}$ is not ig-open in $Y$. Hence $f$ is gi-open.

Theorem2.10. Every continuous mapping is i-continuous.[8][2]

Example2.11. Let $\mathrm{X}=\{\mathrm{s}, \mathrm{t}, \mathrm{u}, \mathrm{v}\}, \tau=\{\phi,\{\mathrm{t}\},\{\mathrm{u}, \mathrm{v}\},\{\mathrm{t}, \mathrm{u}, \mathrm{v}\}, \mathrm{X}\}, \mathrm{Y}=\{\mathrm{x}, \mathrm{y}\}$, $\delta=\{\phi,\{\mathrm{x}\},\{\mathrm{y}\}, \mathrm{Y}\}, f(s)=f(u)=f(v)=x, f(t)=y$.

$\tau^{\mathrm{i}}=\{\phi,\{\mathrm{t}\},\{\mathrm{u}, \mathrm{v}\},\{\mathrm{t}, \mathrm{u}, \mathrm{v}\},\{\mathrm{s}, \mathrm{u}, \mathrm{v}\}, \ldots \ldots \ldots, \mathrm{X}\}, f$ is not continuous because $\{\mathrm{x}\}$ is open set in $Y$ but $f^{-1}(\{\mathrm{x}\})=\{\mathrm{s}, \mathrm{u}, \mathrm{v}\}$ is not open in $X . \quad f$ is icontinuous.

Theorem2.12. Every i-continuous mapping is i-generalized continuous.

Proof: let $f$ be i-continuous mapping and let $\mathrm{G}^{*}$ be closed set in $Y$, then $f^{-1}\left(\mathrm{G}^{*}\right)$ is i-closed in $X$. By (theorem 1.10) we have: $f^{-1}\left(\mathrm{G}^{*}\right)$ is ig-closed in $X$. Hence $f$ is i-generalized continuous.

In (Example 2.5) $f$ is not i-continuous because $\{14\}$ is closed set in $Y$ but $f^{-}$ ${ }^{I}(\{14\})=\{13\}$ is not $\mathrm{i}$-closed in $X . f$ is i-generalized continuous.

Theorem2.13. Every i-generalized continuous mapping is generalized icontinuous.

Proof: Let $f$ be ig-continuous mapping. If $\mathrm{G}^{*}$ is closed set in $Y$ then $f^{-1}\left(\mathrm{G}^{*}\right)$ is ig-closed in $X$. By theorem (1.9) we have: $f^{-1}\left(\mathrm{G}^{*}\right)$ is gi-closed in $X$. Hence $f$ is gi-continuous.

In example 2.8. $f$ is not ig-continuous because $\{\mathrm{q}, \mathrm{r}\}$ is closed $Y$, $f^{l}(\{\mathrm{q}, \mathrm{r}\})=\{\mathrm{p}, \mathrm{r}\}$ is not ig-closed in $X . f$ is gi-continuous.

Theorem2.14. Every topological Homeomorphism is iHomeomorphism.[8][2] 
Example2.15. Let $X=\{g, h, i\}=Y, \tau=\{\phi,\{\mathrm{g}, \mathrm{h}\}, \mathrm{X}\}, \delta=\{\phi,\{\mathrm{g}\}, \mathrm{Y}\}, f(g)=g$, $f(h)=i, f(i)=h, f$ is not continuous because $\{\mathrm{g}\}$ is open set in $\mathrm{Y}, f^{-1}(\{\mathrm{~g}\})=\{\mathrm{g}\}$ is not open in $X . f$ is one-one because $\forall \mathrm{x}_{1 \neq \mathrm{x}_{2}}$ and $f\left(\mathrm{x}_{1}\right) \neq f\left(\mathrm{x}_{2}\right) . f$ is onto because $f(X)=Y$. Hence $f$ is not open because $\{\mathrm{g}, \mathrm{h}\}$ is open in $X$ but $f(\{\mathrm{~g}, \mathrm{~h}\})=\{\mathrm{g}, \mathrm{i}\}$ is not open in $Y$. Hence $f$ is not a Homeomorphism. $\tau^{\mathrm{i}}=\{\phi,\{\mathrm{g}, \mathrm{h}\},\{\mathrm{g}\},\{\mathrm{h}\},\{\mathrm{g}, \mathrm{i}\},\{\mathrm{h}, \mathrm{i}\}, \mathrm{X}\} . \delta^{\mathrm{i}}=\{\phi,\{\mathrm{g}\},\{\mathrm{g}, \mathrm{h}\},\{\mathrm{g}, \mathrm{i}\}, \mathrm{Y}\}$.

$f$ is $\mathbf{i}$-continuous and it is $\mathbf{i}$-open mapping. Hence $f$ is $\mathbf{i}$-Homeomorphism.

Theorem2.16. Every topological i-Homeomorphism is ig-Homeomorphism. Proof: let $f$ be a topological i-Homeomorphism. Then $f$ is (1) i-continuous mapping. , (2) (onto, one-one). (3) i-open mapping. By (theorem 2.12) we have, $f$ is ig-continuous and by corollary (2.6.) we have, $f$ is ig-open. Hence $f$ is ig-Homeomorphism.

In example 2.5. We have, $1 . f$ is not i-open because $\{13,15\}$ is open in $X$ but $f(\{13,15\})=\{14,15\}$ is not $\mathrm{i}$-open in $Y, f$ is ig-open. $2 . f$ is not $\mathrm{i}$ continuous because $\{14\}$ is closed in $Y, f^{-1}(\{14\})=\{13\}$ is not i-closed in $X . f$ is ig-continuous. 3. $f$ is bijective. Therefore, $f$ is ig-homeomorphism is not $\mathrm{i}$ homeomorphism.

Theorem2.17. Every topological ig-Homeomorphism is giHomeomorphism.

Proof: let $f$ be a topological ig-Homeomorphism. We have $f$ is (1) igcontinuous. (2)bijective (onto, one-one)., (3) ig-open. By theorem (2.13) we have, $f$ is gi-continuous mapping and by corollary (2.9.) we have, $f$ is giopen mapping. Hence $f$ is gi-Homeomorphism.

In Example2.8 we have 1. $f$ is not ig-open because $\{\mathrm{p}\}$ is open in $X$ but $f$ $(\{p\})=\{q\}$ is not ig-open in $Y . f$ is gi-open mapping.2. $f$ is not ig-continuous because $\{\mathrm{q}, \mathrm{r}\}$ is closed in $Y$ but $f^{l}(\{\mathrm{q}, \mathrm{r}\})=\{\mathrm{p}, \mathrm{r}\}$ is not ig-closed in $X$. $f$ is gicontinuous . 3. $f$ is bijective. Therefore, $f$ is gi-homeomorphism but is not ig-homeomorphism.

Corollary2.18. Every homeomorphism (respectively i-homeomorphism, ighomeomorphism) is gi-homeomorphism. But the converse is not necessary to be true(theorem (2.14), theorem (2.16) and theorem (2.17)).

Corollary2.19. Every continuous (respectively i-continuous and igcontinuous mapping) is gi-continuous. But the converse is not necessary to be true(theorem (2.10), theorem (2.12) and theorem (2.13)). 
Corollary2.20. Every open (respectively i-open and ig-open mapping) is giopen. But the converse is not necessary to be true(theorem (2.1), corollary (2.6) and corollary (2.9)).

Proposition2.21. For any bijection (onto, one-one) $f$, the following statements are equivalent:

1)Its inverse map $f^{-1}(Y, \sigma) \rightarrow(X, \tau)$ is ig-continuous. 2) $f$ is ig-open. 3) $f$ is ig-closed.

Proposition2.22. Let $f$ be a bijective and ig-continuous map. Then the following statements are equivalent.

1) $f$ is ig-open map.

2) $f$ is ig-homeomorphism.

3) $f$ is ig-closed map.

Proposition2.23. For any bijection (onto, one-one) $f$, the following statements are equivalent:

1)Its inverse map $f^{-1}(Y, \sigma) \rightarrow(X, \tau)$ is gi-continuous. 2) $f$ is gi-open. 3) $f$ is gi-closed.

Proposition2.24. Let $f$ be a bijective and gi-continuous map. Then the following statements are equivalent.

1) $f$ is gi-open map.

2) $f$ is gi-homeomorphism.

3) $f$ is gi-closed map.

Proposition2.25. If a map $f$ is ig-irresolute then, it is ig-continuous.

Proposition2.26. If a map $f$ is gi-irresolute then, it is gi-continuous.

Theorem2.27. A mapping $f:(X, \tau) \rightarrow(Y, \delta)$ is ig-irresolute if and only if the inverse of $I^{*}$ is ig-open set in $X$ for all ig-open sets $I^{*}$ in $Y$.

Proof. Suppose that $\mathrm{f}$ is ig-irresolute mapping.

Let $I^{*}$ be ig-open set in Y. then $I^{* c}$ is ig-closed set in $Y$. we have, $f^{-1}\left(\left(I^{*}\right)^{c}\right)$ is ig-closed set in $\mathrm{X}$ (by suppose). We have, $\left(f^{-1}\left(I^{*}\right)\right)^{c}$ is ig-closed set in $\mathrm{X}$. Hence $f^{-1}\left(I^{*}\right)$ is ig-open set in $\mathrm{X}$. 
Conversely, suppose that the inverse of every ig-open set in $Y$ is ig-open in $X$. Let $I^{*}$ be ig-closed set in Y. then $I^{* c}$ is ig-open in $Y$. we have, $f^{-l}\left(\left(I^{*}\right)^{c}\right)$ is ig-open set in $\mathrm{X}$ (by suppose). We have, $\left(f^{-1}\left(I^{*}\right)\right)^{c}$ is ig-open set in $\mathrm{X}$. Hence $f^{-1}\left(I^{*}\right)$ is ig-closed set in X. Therefore, $\mathrm{f}$ is ig-irresolute map.

Theorem2.28. A mapping $f:(X, \tau) \rightarrow(Y, \delta)$ is gi-irresolute if and only if the inverse of $I^{*}$ is gi-open set in $X$ for all gi-open sets $I^{*}$ in $Y$.

Proof. Suppose that $\mathrm{f}$ is gi-irresolute mapping.

Let $I^{*}$ be gi-open set in Y. then $I^{*_{c}}$ is gi-closed in Y. we have, $f^{-1}\left(\left(I^{*}\right)^{c}\right)$ is gi-closed in $\mathrm{X}$ (by suppose). We have, $\left(f^{-1}\left(I^{*}\right)\right)^{c}$ is gi-closed in $\mathrm{X}$. Hence $f^{-1}\left(I^{*}\right)$ is gi-open set in X.

Conversely, suppose that the inverse of every gi-open set in $Y$ is gi-open in $X$. Let $I^{*}$ be gi-closed set in $\mathrm{Y}$. then $I^{* c}$ is gi-open in $Y$. we have, $f^{-1}\left(\left(I^{*}\right)^{c}\right)$ is gi-open set in $\mathrm{X}$ (by suppose). We have, $\left(f^{-1}\left(I^{*}\right)\right)^{c}$ is gi-open in $\mathrm{X}$. Hence $f^{-1}\left(I^{*}\right)$ is gi-closed set in $\mathrm{X}$. Therefore, $\mathrm{f}$ is gi-irresolute map.

Theorem2.29. Every i-generalized irresolute mapping is generalized iirresolute.

Proof: Let $f$ be ig-irresolute mapping. If $\mathrm{G}^{*}$ is ig-closed set in $Y$ then $f^{-1}\left(\mathrm{G}^{*}\right)$ is ig-closed in $X$. By theorem (1.9) we have: $f^{-1}\left(\mathrm{G}^{*}\right)$ is gi-closed in $X$. Hence $f$ is gi-irresolute.

In example 2.8. $f$ is not ig-irresolute because $\{\mathrm{q}, \mathrm{r}\}$ is ig-closed set in $Y, f^{1}$ $(\{\mathrm{q}, \mathrm{r}\})=\{\mathrm{p}, \mathrm{r}\}$ is not ig-closed in $X . f$ is not gi-irresolute because $\{\mathrm{q}\}$ is giclosed set in $Y, f^{1}(\{q\})=\{p\}$. is not gi-closed in $X$.

\section{References}

[1] Arya , S. P. and Nour, T. "Characterizations of s-normal spaces" Indian J. Pure appl. Math. 21(8)(1990),717-719.

[2] Askandar, S.W., M.Sc. Thesis, College of Education, University of Mosul (2012). (In Arabic).

[3] Bhattacharyya, P. and Lahiri, B. K. "Semi Generalized Closed Sets in Topology." Indian J. Math 29 (1987), 376- 82.

[4]Caldas, M., Jafari, S. and Rajesh, N., " On $\tilde{g}$-Homeomorphisms in Topological Spaces" Universidad Cat'olica del Norte Antofagasta - Chile, Vol. 28, No 1, pp. 1-19, May 2009.

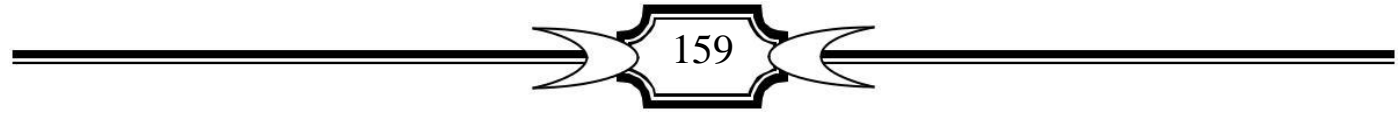


[5] Devi, R., Maki, H. and Balachandran, K. "Semi -Generalized Homeomorphism and Generalized semi-Homeomorphism in topological spaces" Indian J. Pure appl. Math26 (3)(1995),271-284.

[6] Jardo, M.A., M.Sc. Thesis, College of Education, University of Mosul (2013). (In Arabic).

[7] Levine, N. "Generalized closed sets in topology" Rend Circ. Mat. Patemo (2) 19 (1970), 89-96.

[8]Mohammed, A.A. and Askandar, S.W., On i-open sets, UAE Math Day Conference, American Univ. of Sharjah, April 14, 2012.

[9] Rajesh, N., Ekici, E. and Thivagar, M. L. " On $\tilde{g}$-SemiHomeomorphism in Topological Spaces" Annals of University of Craiova, Math. Comp. Sci. Ser.Volume 33, 2006, Pages 208-215, ISSN: 1223-6934. 